\title{
Are all radio galaxies genuine ellipticals?^
}

\author{
M. P. Véron-Cetty ${ }^{1}$ and P. Véron ${ }^{1}$ \\ Observatoire de Haute Provence, CNRS, 04870 Saint-Michel l'Observatoire, France \\ e-mail: mira@obs-hp.fr \\ Received 16 January 2001 / Accepted 11 June 2001

\begin{abstract}
Classical double radio sources are believed to be powered by a strong relativistic jet due to the presence of a rapidly spinning black hole in the center of a giant $\mathrm{E}$ galaxy formed by the merging of two galaxies. If this is true, no radio source should have been found in spiral or S0 galaxies. A number of radio S0s have been reported, but most of them are probably misclassified Es. However, our own observations confirm that NGC 612 is an S0 although it is associated with the FR II radio source PKS 0131-36. We conclude that S0s can be classical radio sources, but that such occurences are extremely rare.
\end{abstract}

Key words. galaxies: elliptical and lenticular, cD - galaxies: individual: NGC 612 - radio continuum: galaxies

\section{Introduction}

Most classical double radio sources (FR Is and FR IIs; Fanaroff \& Riley 1974) are associated with giant elliptical (E) galaxies. It has been suggested that these radio sources are the result of the presence in the nucleus of the galaxies of a rapidly spinning black hole and that these black holes are due to the merging of two galaxies implying that S0s and spirals cannot be the host of a double radio source. About two dozen radio galaxies have been classified as S0s, but early-type galaxies are difficult to classify and we show that most of them have been misclassified; however NGC 612 is an FR II radio galaxy and is a bona fide $\mathrm{S} 0$.

\section{2. $\mathrm{E}$ and $\mathrm{SO}$ galaxies}

The difference between $\mathrm{E}$ and $\mathrm{S} 0$ galaxies resides in the presence or absence of a disk. Es, by definition, have no disk; S0s, by definition, do (Sandage \& Bedke 1994).

De Vaucouleurs (1948) showed that the brightness distribution of $\mathrm{E}$ galaxies can be represented by the socalled $r^{1 / 4}$ law:

$\log (I)=\log \left(I_{\mathrm{e}}\right)-3.331 \times\left[\left(r / r_{\mathrm{e}}\right)^{1 / 4}-1\right]$

where $I$ is the surface brightness at radius $r, r_{\mathrm{e}}$ the effective radius containing half of the light of the model and $I_{\mathrm{e}}$ the effective surface brightness at that radius. $r_{\mathrm{e}}$ varies from $\approx 1.0$ to $50 \mathrm{kpc}^{1}$ (Hamabe \& Kormendy 1987; Ledlow \& Owen 1995).

Send offprint requests to: P. Véron,

e-mail: veron@obs-hp.fr

* Partly based on observations obtained with the ESO $3.6 \mathrm{~m}$ telescope, La Silla, Chile.

${ }^{1} H_{0}=50 \mathrm{~km} \mathrm{~s}^{-1} \mathrm{Mpc}^{-1}$ is assumed throughout this paper.
S0 light profiles can be separated into two components: (1) a bulge component characterized by an E-like surface brightness distribution, and (2) a disk component with an exponential light distribution of the form:

$I=I_{\mathrm{o}} \times \mathrm{e}^{-r / r_{\mathrm{o}}}$

where $r_{\mathrm{o}}$ is the scale length of the exponential disk (Freeman 1970).

\section{Dichotomy between bright and weak ellipticals}

Bright Es have a markedly different distribution of Hubble types than faint Es, the division occuring near $M_{B}=-20$; brighter Es have Hubble types that are narrowly clustered around E1-E2, while fainter Es have Hubble types near E3 (Jaffe et al. 1994; Tremblay \& Merritt 1996). Isophotes of E galaxies often show systematic deviations from ellipticity; some Es have box-shaped or "boxy" isophotes, while others have disk-shaped or "disky" isophotes (Carter 1978; Lauer 1985). Disky isophotes can be attributed to the presence of a weak, more or less edge-on, disk superposed on a spheroidal main body (Bender et al. 1987). Jaffe et al. (1994) found that all Es fainter than $M_{B}=-20$ are disky.

Disky and faint Es $\left(M_{B}>-20.8\right)$ are in general consistent with being rotationally flattened; in contrast, boxy and bright Es are slow rotators (Davies et al. 1983; Carter 1987; Bender 1988; Capaccioli \& Longo 1994).

At small radii, E galaxies have two types of brightness profiles. The first type consists of galaxies that have a cuspy core; these galaxies have brightness profiles that break from steep outer power laws to shallow inner cusps. The second type consists of galaxies that have profiles that continue into the resolution limit as steep power laws, 
showing no evidence of cores (Lauer et al. 1995; Gebhardt et al. 1996). Luminous $\left(M_{B}<-21.0\right)$ and boxy Es have a cuspy core; faint $\left(M_{B}>-19.5\right)$ and disky Es show powerlaw profiles; it is possible that all power-law Es are in fact S0s masquerading as ellipticals (Nieto et al. 1991; Faber et al. 1997; Michard 1998b).

"Bright" galaxies are characterized by very large effective radii $\left.\left(<r_{\mathrm{e}}\right\rangle=10.5 \mathrm{kpc}\right)$, while "ordinary" galaxies do not grow larger in size than $r_{\mathrm{e}}=3 \mathrm{kpc}$ (D'Onofrio et al. 1994).

Based on these facts, Capaccioli et al. (1992), Kormendy \& Bender (1996) and Kormendy (1999) proposed that spirals, S0s and low luminosity, disky Es form a continuous sequence while giant, boxy Es are unrelated to other ellipticals.

A number of observational facts indicate that giant Es are the result of merging (Merritt 1993; Capaccioli \& Longo 1994). In particular, massive boxy Es present signatures of merging processes (Nieto \& Bender 1989). For Naab et al. (1999), merging of two equal-mass spirals leads to an anisotropic, slowly rotating system with preferentially boxy isophotes, while unequal-mass mergers result in the formation of rotationally supported Es with disky isophotes.

\section{Host galaxies of double radio sources}

\subsection{Radio source host galaxies as giant Es}

A number of pieces of observational evidence suggest that most radio source host galaxies are giant Es (Lilly \& Prestage 1987; Owen \& Laing 1989; Owen \& White 1991; Govoni et al. 2000a; Hamilton et al. 2000) with scalelengths in the range 6-23 kpc (McLure et al. 1999).

Low luminosity classical radio galaxies (FR Is) are luminous Es showing evidence for a well resolved, shallow central cusp when they are not severely affected by dust extinction, suggesting that they belong to the class of "bright" Es (Verdoes Kleijn et al. 2000; Capetti et al. 2000).

A large fraction of both FR Is (Colina \& de Juan 1995) and FR IIs (Smith \& Heckmam 1989b; Hutchings 1987) radio galaxies exhibit peculiar optical morphologies (tails, fans, bridges, shells, isophote twists, nonconcentric isophotes, filaments) which are probably products of galaxy interaction; a significant number are optically interacting.

Large amounts $\left(10^{9}\right.$ to $\left.10^{11} M_{\odot}\right)$ of ${ }^{12} \mathrm{CO}$ have been detected in a number of radio galaxies; mergers of two gasrich disk galaxies provide an attractive explanation for the presence of this gas (Lim et al. 2000).

\subsection{Do radio-loud $S 0$ galaxies exist?}

The radio detection rate for S0s is very low compared with that for Es, with the possibility that the radio-loud S0s are misclassified Es (Ekers \& Ekers 1973; Dressel 1981; Hummel \& Kotanyi 1982). Early-type galaxies are indeed difficult to classify; a relatively large fraction (up to 30\%) of early-type galaxies have a different classification in different catalogues (Jenkins 1982; Bregman et al. 1992).

Morphological classification of dust lane galaxies have, for a long time, been subject to uncertainty; according to Sandage (1961) there is no optical evidence for dust in Es; consequently, early-type galaxies with dust have, in the past, been given S0 classifications (Kotanyi \& Ekers 1979; Hawarden et al. 1981; Ebneter et al. 1988). In fact, even the classical Es often contain dust (Goudfrooij et al. 1994), although dust features appear to be much more frequent in disky than in boxy Es (Michard 1998a).

cDs are extreme giant Es which have a separate extended, low surface brightness envelope (Tonry 1987; Schombert 1988). As a consequence, a number of $\mathrm{cD}$ radio galaxies have been erroneously classified as S0s (Matthews et al. 1964).

Twenty three classical double radio galaxies (either FR Is or FR IIs) classified as disk systems have been mentioned in the literature; they are listed in Table 1 together with NGC 612.

The analysis of the morphology of these galaxies shows that most of them were misclassified as S0s, because either they are cDs, or they contain dust, or their morphology is disturbed by interaction with another closeby galaxy. In a few cases, the classification is doubtful due to a lack of high quality observations.

Four radio galaxies classified as S0s are probably cDs instead: 3C 29.0 (Fasano et al. 1996), NGC 383 (Michard 2001, private communication), NGC 547 (Fasano et al. 1996) and NGC 1316 (Schweizer 1980).

Three Es have been misclassified as S0s, probably because of the presence of dust: UGC 367 (González-Serrano \& Pérez-Fournon 1991), 3C 223.1 (de Koff et al. 2000) and 3C 268.2 (Hutchings et al. 1988; de Koff et al. 2000).

Several Es have disturbed isophotes due to interaction with a close companion or a recent merging: 3C 136.1 (Martel et al. 1999; de Koff et al. 2000), NGC 5128 (Malin et al. 1983), NGC 5141 (González-Serrano et al. 1993), MCG 05.33.12 (Heckman et al. 1986; Evans et al. 1999), IC 1065 (Heckman et al. 1982; de Koff et al. 2000), NGC 6047 (Heckman et al. 1986) and NGC 7237 (Borne \& Hoessel 1988; Comins \& Owen 1991).

In a few cases, the early S0 classification was based on poor scale photographs; more recent, better quality observations show that the object is really an elliptical: PKS 0449-17 (Fasano et al. 1996), 3C 223.0 (Hutchings et al. 1988; de Koff et al. 1996), NGC 4789 (Bender et al. 1989; de Juan et al. 1994) and NGC 5532 (Bender et al. 1989; de Juan et al. 1994).

In two cases (PKS 1053-282 and PKS 1344-241) the radio morphology is poorly known and it is possible that the radio source is not a classical double radio source (Ekers et al. 1989).

The available data are sometimes insufficient to conclude, e.g. PKS 0257-398 (Govoni et al. 2000b) and 3C 89.0 (Smith \& Heckman 1989a; Zirbel 1996). 
Table 1. List of classical double radio sources mentioned in the literature as being S0s.

\begin{tabular}{llll}
\hline Position & Optical name & Radio name & $z$ \\
\hline $0034+25$ & UGC 367 & B2 0034+25 & 0.032 \\
$0055-01$ & UGC 595 & 3C 29.0 & 0.045 \\
$0104+32$ & NGC 383 & 3C 31.0 & 0.017 \\
$0123-01$ & NGC 547 & 3C 40.0 & 0.018 \\
$0130-36$ & NGC 612 & PKS 0131-36 & 0.030 \\
$0257-39$ & & PKS 0257-398 & 0.061 \\
$0320-37$ & NGC 1316 & PKS 0320-37 & 0.006 \\
$0331-01$ & & 3C 89.0 & $0.139 ?$ \\
$0449-17$ & ESO 552-G14 & PKS 0449-17 & 0.032 \\
$0512+24$ & & 3C 136.1 & 0.064 \\
$0936+36$ & & 3C 223.0 & 0.137 \\
$0938+39$ & & 3C 223.1 & 0.107 \\
$1053-28$ & & PKS 1053-282 & 0.061 \\
$1122+39$ & NGC 3665 & B2 1122+39 & 0.007 \\
$1158+31$ & & 3C 268.2 & 0.361 \\
$1251+27$ & NGC 4789 & 5C 4.20 & 0.028 \\
$1322-42$ & NGC 5128 & PKS 1322-42 & 0.002 \\
$1322+36$ & NGC 5141 & B2 1322+36 & 0.018 \\
$1344-24$ & ESO 509-G108 & PKS 1344-241 & 0.020 \\
$1350+31$ & MCG 05.33.12 & 3C 293.0 & 0.045 \\
$1414+11$ & NGC 5532 & 3C 296.0 & 0.024 \\
$1448+63$ & IC 1065 & 3C 305.0 & 0.041 \\
$1602+17$ & NGC 6047 & PKS 1602+178 & 0.032 \\
$2212+13$ & NGC 7237 & 3C 442.0 & 0.026 \\
\hline
\end{tabular}

The true nature of NGC 3665 is rather controversial (Davies \& Birkinshaw 1988; Peletier et al. 1990; Burkert 1993; Michard \& Marchal 1994).

\subsection{The case of NGC 612}

PKS 0131-36 has been identified with the 14.2 mag galaxy NGC 612 (Westerlund \& Smith 1966). A weak radio core detected by Morganti et al. (1993) coincides, within the measurement errors $\left( \pm 00^{\prime \prime} 5\right)$, with the optical nucleus of the galaxy (Jones \& McAdam 1992), making the reality of the identification unquestionable.

A radio map published by Morganti et al. (1993) shows it to be a typical FR II radio source. With a $1400 \mathrm{MHz}$ flux density of $7.1 \mathrm{Jy}$, and a redshift $z=0.030$, the absolute luminosity is $P_{1400}=2.6 \times 10^{25} \mathrm{~W} \mathrm{~Hz}^{-1}$; assuming $i=11.42$ (see below), and $R-i=0.20$ for an E galaxy (Véron-Cetty \& Woltjer 1990), the absolute $R$ magnitude of the galaxy within an isophote of $24.5 \mathrm{mag} \operatorname{arcsec}^{-2}$ is -23.9. If $H_{0}=75 \mathrm{~km} \mathrm{~s}^{-1} \mathrm{Mpc}^{-1}$, these numbers become $P_{1400}=1.2 \times 10^{25} \mathrm{~W} \mathrm{~Hz}^{-1}$ and $M_{R}=-23.0$; thus, according to Ledlow \& Owen (1996), this object would be classified as an FR I, but near the borderline between FR Is and FR IIs.

NGC 612 has a fairly well-developped luminous disk seen almost edge-on (Kotanyi \& Ekers 1979) with a strong dust ring (Westerlund \& Smith 1966; Fasano et al. 1996). It was classified as an S0 by Kotanyi \& Ekers and Sansom et al. (1987) and as an S0-a by Lauberts (1982); Westerlund \& Smith (1966) described it as an S0-a peculiar galaxy. Goss et al. (1980) showed that the galaxy is rotating, the dust ring being orthogonal to the rotation axis of the galaxy. However, according to Fasano et al. (1996), the luminosity profile follows the de Vaucouleurs law almost perfectly with an effective radius $r_{\mathrm{e}}=16.72 \mathrm{kpc}$ (assuming $H_{0}=50 \mathrm{~km} \mathrm{~s}^{-1} \mathrm{Mpc}^{-1}$ ).

We have obtained, on August 15, 1993, three $90 \mathrm{~s}$ images of NGC 612 through a Gunn $i$ filter (7200-8500 A) (seeing 1".5) and, on the next day, one $10 \mathrm{~min}$ image through a $B$ filter (seeing $1^{\prime \prime} 6$ ), at the ESO $3.6 \mathrm{~m}$ telescope at La Silla, with EFOSC and a $512 \times 51227 \mu \mathrm{m}$ pixel (0.'607) Tektronix CCD. Figure 1 shows the sum of the three $i$ images.

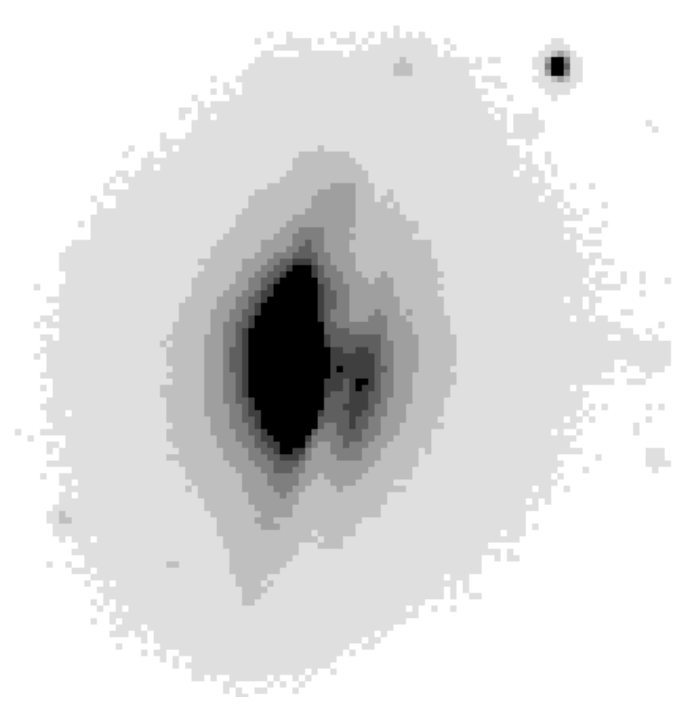

Fig. 1. $i$ image of NGC 612 . The total exposure time is $270 \mathrm{~s}$. North is to the top, East to the left; the size of the image is $67 \times 73$ arcsec.

Figure 2 is the ratio between the $B$ and $i$ images and shows the dust feature which appears as two parallel lanes.

The programme PLEINPOT written by P. Prugniel, which fits ellipses over the whole galaxy, was used to analyse the $i$ image; the centre of the galaxy was found $1 . \prime 2 \mathrm{~W}$ and $0^{\prime \prime} \cdot 2 \mathrm{~N}$ of the maximum intensity (because of the strong dust feature obscuring the nucleus); the galaxy has an axis ratio $b / a=0.75$ at $\mathrm{PA} 168^{\circ}$, in agreement with Ekers et al. (1978).

The mean surface brightness was measured in ellipses having an axis ratio equal to 0.75 at $\mathrm{PA} 168^{\circ}$. An $r^{1 / 4}$ fit to the surface brightness profile of the $i$ image is not satisfactory; adding a disk, we obtain (Fig. 3):

a) for the $r^{1 / 4}$ component: $r_{\mathrm{e}}=17^{\prime \prime} .7(15.5 \mathrm{kpc})$ (in good agreement with the value found by Fasano et al. (1996), integrated $i$ magnitude $=11.83$ (assuming $i=$ 12.31 for the standard star LTT 3218; Véron-Cetty \& Woltjer 1997);

b) for the disk: $r_{\mathrm{o}}=118^{\prime \prime} 2$, integrated $i$ magnitude $=12.68$.

The integrated $i$ magnitude for the model is 11.42 . 


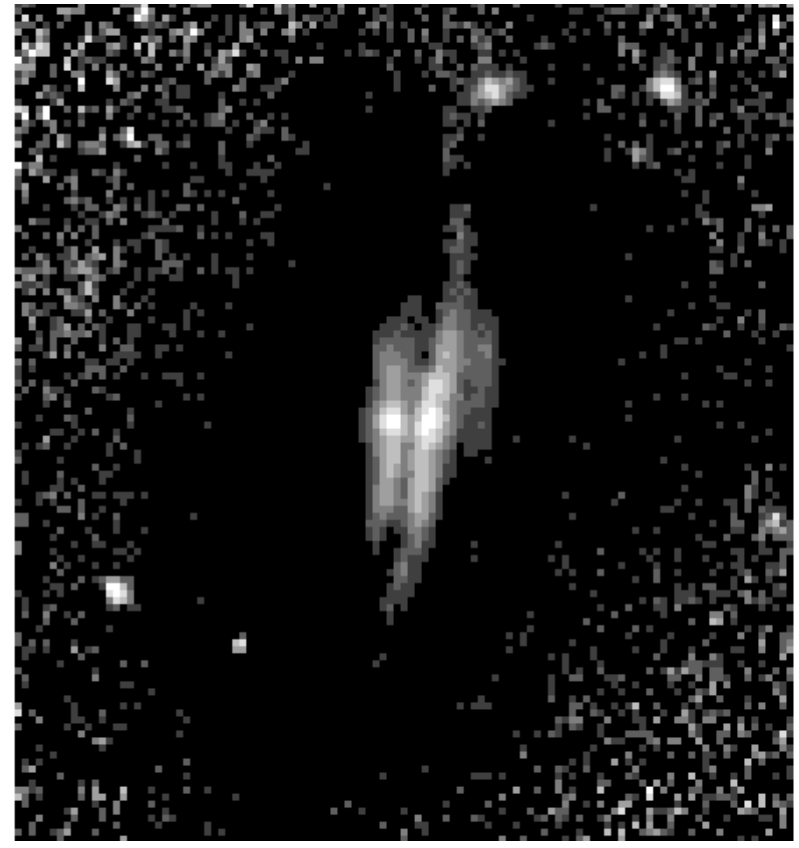

Fig. 2. Ratio of the $B$ to the $i$ images showing the distribution of the dust. The orientation and size are the same as in Fig. 1.

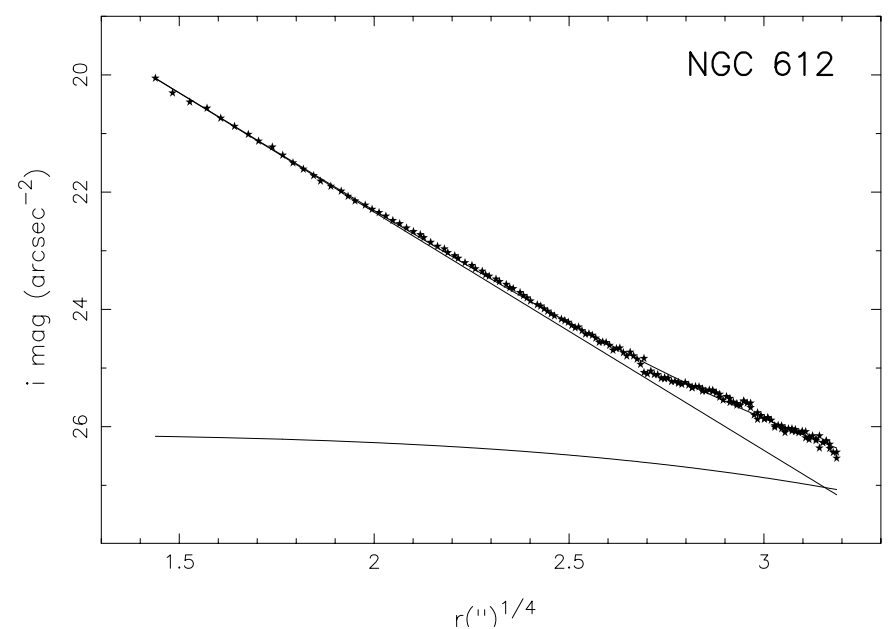

Fig. 3. $i$ brightness profile of NGC 612 and fit obtained with a two component model.

This does not contradict the abovementioned result of Fasano et al. (1996), as these authors do not go beyond $r^{1 / 4}=2.8$.

The fit of the $B$ image is meaningless because of the strong absorption lane.

Figure 4 is the result of the subtraction from the $i$ image of a E model galaxy having the parameters deduced from the fit. This image shows the disk of the galaxy strongly perturbed by the dust.

Ekers et al. (1978) remarked that NGC 612 was the only known radio galaxy with an obvious stellar disk, but their conclusion was based on the examination of photographic plates; we confirm, with CCD images obtained with a larger telescope, that this galaxy is indeed an S0.

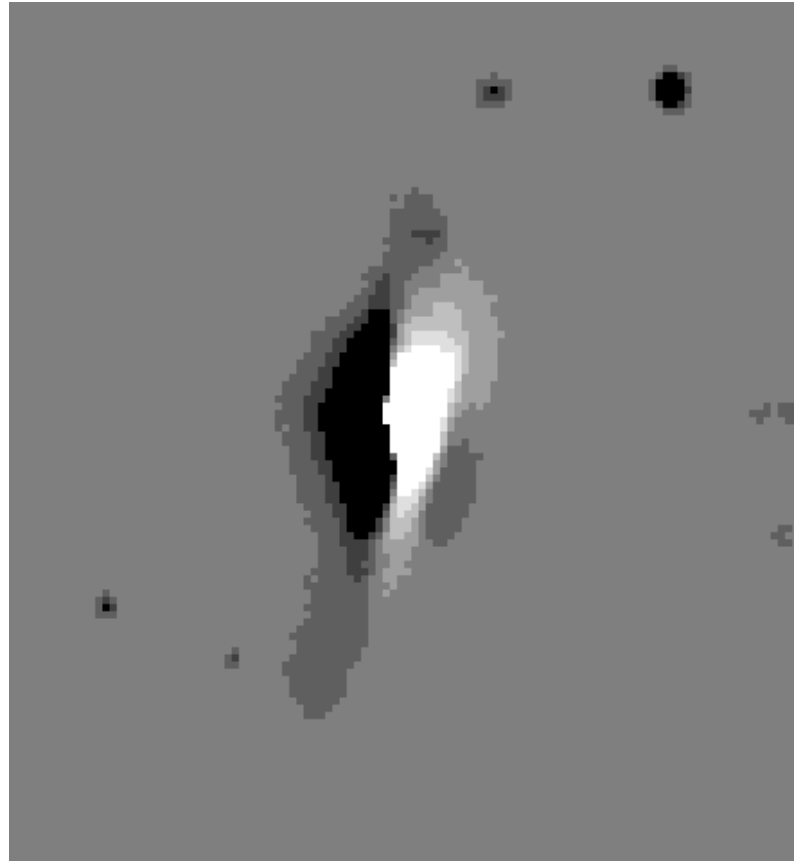

Fig. 4. Result of the subtraction from the $i$ image of an model E galaxy which is centered $1^{\prime \prime} .2 \mathrm{~W}$ of the $i$ peak intensity, has an $r^{1 / 4}$ profile with $r_{\mathrm{e}}=17^{\prime \prime} .7$, and $b / a=0.75$ at $\mathrm{PA} 168^{\circ}$ and is convolved with a PSF). The orientation and size are the same as in Fig. 1.

\subsection{Classical double radio sources as spiral galaxies?}

Three radio sources have, in the past, been reported to be associated with a spiral galaxy: NGC 5972, PKS 0400-181, and IRAS 04210+0400. Although NGC 5972 was classified as an S0-a by Nilson (1973), Véron \& Véron-Cetty (1995) showed that it is an E with tidal arms. PKS $0400-181$ was first identified with a spiral galaxy (Savage \& Wall 1976), but Shaver et al. (1983) showed that the true identification is an E at $z=0.341$ located behind the spiral. Beichman et al. (1985) reported the discovery of a type 2 Seyfert nucleus (IRAS $04210+0400$ ) in what appears to be a spiral galaxy associated with a double lobed radio source; Hill et al. (1988) showed that the radio source is an FR I but that there is no evidence that the galaxy is a spiral.

Gonzáles-Serrano \& Carballo (2000) have analysed CCD images of 70 galaxies associated with a low luminosity B2 radio source; they classified 57 galaxies as Es, seven as irregulars and six as spirals; five of these last six radio sources are unrelated to the spiral galaxies with which they had initially been identified on the basis of rather poor radio positions. The identification of the sixth object, B2 $0722+30$, with $\mathrm{Z}_{\mathrm{w}} 147.020$ was confirmed by accurate radio positions; the galaxy is a spiral, but both the size $(9 \mathrm{kpc})$ and the luminosity of the radio source are typical of radio sources associated with spiral Seyfert galaxies (Capetti et al. 2000).

However, Ledlow et al. (2001) have reported the discovery of an FR I radio source associated with a spiral galaxy in the cluster Abell 428. Moreover, the BL Lac 
object PKS $1413+135$ is hosted by a spiral (Lamer et al. 1999); BL Lac objects are supposed to be FR I radio sources with the radio jet oriented towards the observer. So it seems now well established that, although extremely rare, double radio sources associated with a spiral galaxy do exist.

\section{Discussion}

There is a very limited range of inputs that determine the nature of an AGN: the fuel supply, the mass of the black hole (BH), and its angular momentum. The fuel supply rate is similar for AGNs in radio-loud and radio-quiet objects since both cover the whole range of luminosities; regarding the mass of the $\mathrm{BH}$, the Eddington limit indicates that masses up to $10^{8} M_{\odot}$ occur in some radio-quiet AGNs; somewhat more massive BHs could occur in radio galaxies, but masses in excess of $10^{10} M_{\odot}$ seem to be ruled out on dynamical grounds, at any rate in the nearest Es; the difference between radio-loud and radioquiet galaxies should then be the larger angular momentum of the central BH in radio-loud objects (Blandford 1990; Scheuer 1992; Meier 1999). However, according to Laor (2000), when the mass of the central $\mathrm{BH}$ is larger than $10^{9} M_{\odot}$, the object is radio-loud while, when it is smaller than $3 \times 10^{8} M_{\odot}$, the object is radio-quiet. Blandford \& Znajek (1977) showed that rotational energy can be extracted from a spinning $\mathrm{BH}$ surrounded by a magnetized accretion disk. The BZ mechanism seems to be adequate to produce the radio jet in FR II radio sources in which the $\mathrm{BH}$ is accreting at or near the Eddington rate (Moderski \& Sikora 1996). In the case of FR I radio sources which are probably accreting at sub-Eddington rates, the question is controversial (Rees et al. 1982; Ghosh \& Abramowicz 1997; Livio et al. 1999).

Blandford (1990) argued that sub-Eddington mass accretion rates produce slowly spinning $\mathrm{BHs}$ and that rapidly spinning $\mathrm{BHs}$ require high accretion rates. However, accretion from a disk is an ineffective way to spin up BHs since little of the disk's angular momentum falls into the hole; on the other hand, two BHs of comparable mass can spiral together to produce a rapidly-spinning hole (Wilson \& Colbert 1995). Moreover, in FR Is objects, the accretion rates are certainly small and yet these objects have a radio jet implying a rapidly rotating $\mathrm{BH}$, while narrow line Seyfert 1s are generally radio-quiet spiral galaxies which are believed to contain a small mass $\mathrm{BH}$ accreting matter at a high rate. Therefore, the larger angular momentum of the central $\mathrm{BH}$ in radio loud $\mathrm{E}$ galaxies is more probably the result of the merging of two BHs (Scheuer 1992).

It seems therefore that classical double radio sources which are characterized by the presence of a radio jet should be associated with bright, boxy Es resulting from the merging of two spiral galaxies, each containing a nuclear $\mathrm{BH}$ of similar mass. It seems however that this is not always the case as, as we have seen, radio-loud spiral and S0 galaxies do exist.

\section{Conclusion}

Most classical double radio sources are hosted by giant E galaxies. This led to a scenario in which these sources, which are powered by a strong relativistic jet, are due to the presence of a rapidly spinning $\mathrm{BH}$ in the center of an E galaxy resulting from the merging of two galaxies. However, although extremely rare, S0 and spiral hosts exist which cannot be explained by this mechanism.

Acknowledgements. We would like to thank R. Michard for useful advices and P. Prugniel for allowing us to use his program PLEINPOT.

\section{References}

Beichman, C., Wynn-Williams, C. G., Lonsdale, C. J., et al. 1985, ApJ, 293, 148

Bender, R. 1988, A\&A, 193, L7

Bender, R., Döbereiner, S., \& Möllenhoff, C. 1987, A\&AS, 74, 385

Bender, R., Surma, P., Döbereiner, S., \& Möllenhoff, C., \& Madejsky, R. 1989, A\&A, 217, 35

Blandford, R. D. 1990, in Active galactic nuclei, Saas-Fee advanced course 20, ed. T. J.-L. Courvoisier, \& M. Mayor, 161

Blandford, R. D., \& Znajek, R. L. 1977, MNRAS, 179, 433

Borne, K. D., \& Hoessel, J. G. 1988, ApJ, 330, 51

Bregman, J. N., Hogg, D. E., \& Roberts, M. S. 1992, ApJ, 387, 484

Burkert, A. 1993, A\&A, 278, 23

Capaccioli, M., \& Longo, G. 1994, A\&AR, 5, 293

Capaccioli, M., Caon, N., \& D'Onofrio, M. 1992, MNRAS, 259, 323

Capetti, A., de Ruiter, H. R., Fanti, R., et al. 2000, A\&A, 362, 871

Carter, D. 1978, MNRAS, 182, 797

Carter, D. 1987, ApJ, 312, 514

Colina, L., \& de Juan, L. 1995, ApJ, 448, 548

Comins, N. F., \& Owen, F. N. 1991, ApJ, 382, 108

Davies, R. L., \& Birkinshaw, M. 1988, ApJS, 68, 409

Davies, R. L., Efstathiou, G., Fall, S. M., Illingworth, G., \& Schechter, P. L. 1983, ApJ, 266, 41

de Koff, S., Baum, S. A., Sparks, W. B., et al. 1996, ApJS, 107, 621

de Koff, S., Best, P., Baum, S. A., et al. 2000, ApJS, 129, 33

de Juan, L., Colina, L., \& Pérez-Fournon, I. 1994, ApJS, 91, 507

de Vaucouleurs, G. 1948, Ann. Astrophys., 11, 247

D'Onofrio, M., Capaccioli, M., \& Caon, N. 1994, MNRAS, 271, 523

Dressel, L. L. 1981, ApJ, 245, 25

Ebneter, K., Djorgovski, S., \& Davies, M. 1988, AJ, 95, 422

Ekers, R. D., \& Ekers, J. A. 1973, A\&A, 24, 247

Ekers, R. D., Goss, W. M., Kotanyi, C. G., \& Skellern, D. J. 1978, A\&A, 69, L21

Ekers, R. D., Wall, J. V., Shaver, P. A., et al. 1989, MNRAS, 236, 737

Evans, A. S., Sanders, D. B., Surace, J. A., \& Mazzarella, J. M. 1999, ApJ, 511, 730

Faber, S. M., Tremaine, S., Ajhar, E. A., et al. 1997, AJ, 114, 1771

Fanaroff, B. L., \& Riley, J. M. 1974, MNRAS, 167, 31P 
Fasano, G., Falomo, R., \& Scarpa, R. 1996, MNRAS, 282, 40 Freeman, K. C. 1970, ApJ, 160, 811

Gebhardt, K., Richstone, D., Ajhar, E. A., et al. 1996, AJ, 112, 105

Ghosh, P., \& Abramowicz, M. A. 1997, MNRAS, 292, 887

González-Serrano, J. I., \& Pérez-Fournon, I. 1991, A\&A, 249, 75

González-Serrano, J. I., \& Carballo, R. 2000, A\&AS, 142, 353

González-Serrano, J. I., Carballo, R., \& Pérez-Fournon, I. 1993, AJ, 105, 1710

Goss, W. J. M., Danziger, I. J., Forbury, R. A. E., \& Boksenberg, A. 1980, MNRAS, 190, 23P

Goudfrooij, P., Hansen, L., Jørgensen, H. E., \& NørgaardNielsen, H. U. 1994, A\&AS, 105, 341

Govoni, F., Falomo, R., Fasano, G., \& Scarpa, R. 2000a, A\&A, 353,507

Govoni, F., Falomo, R., Fasano, G., \& Scarpa, R. 2000b, A\&AS, 143, 369

Hamabe, M., \& Kormendy, J. 1987, IAU Symp., 127, 379

Hamilton, T. S., Casertano, S., \& Turnshek, D. A. 2000 [astro-ph/0011255]

Hawarden, T. G., Elson, R. A. W., Longmore, A. J., Tritton, S. B., \& Corwin, Jr. H. G. 1981, MNRAS, 196, 747

Heckman, T. M., Miley, G. K., Balick, B., van Breugel, W. J. M., \& Butcher, H. R. 1982, ApJ, 262, 529

Heckman, T. M., Smith, E. P., Baum, S. A., et al. 1986, ApJ, 311,526

Hill, G. J., Wynn-Williams, C. G., Becklin, E. E., \& MacKenty, J. W. 1988, ApJ, 335, 93

Hummel, E., \& Kotanyi, C. G. 1982, A\&A, 106, 183

Hutchings, J. B. 1987, ApJ, 320, 122

Hutchings, J. B., Johnson, I., \& Pyke, R. 1988, ApJS, 66, 361

Jaffe, W., Ford, H. C., O'Connell, R. W., van den Bosch, F. C., \& Ferrarese, L. 1994, AJ, 108, 1567

Jenkins, C. R. 1982, MNRAS, 200, 705

Jones, P. A., \& McAdam, W. B. 1992, ApJS, 80, 137

Kormendy, J. 1999, ASP Conf. Ser., 182, 124

Kormendy, J., \& Bender, R. 1996, ApJ, 464, L119

Kotanyi, C. G., \& Ekers, R. D. 1979, A\&A, 73, L1

Lamer, G., Newsam, A. M., \& McHardy, I. M. 1999, MNRAS, 309, 1085

Laor, A. 2000, ApJ, 543, L111

Lauberts, A. 1982, The ESO/Uppsala Survey of the ESO(B) Atlas, European Southern Observatory, Garching bei München

Lauer, T. R. 1985, MNRAS, 216, 429

Lauer, T. R., Ajhar, E. A., Byun, Y.-I., et al. 1995, AJ, 110, 2622

Ledlow, M. J., \& Owen, F. N. 1995, AJ, 110, 1959

Ledlow, M. J., \& Owen, F. N. 1996, AJ, 112, 9

Ledlow, M. J., Owen, F. N., Yun, M. S., \& Hill, J. M. 2001, ApJ, 552, 120

Lilly, S. J., \& Prestage, R. M. 1987, MNRAS, 225, 331

Lim, J., Leon, S., Combes, F., \& Dinh-V-Trung 2000, ApJ, 545, L93

Livio, M., Ogilvie, G. I., \& Pringle, J. E. 1999, ApJ, 512, 100
Malin, D. F., Quinn, P. J., \& Graham, J. A. 1983, ApJ, 272, L5

Martel, A. R., Baum, S. A., Sparks, W. B., et al. 1999, ApJS, 122,81

Matthews, T. A., Morgan, W. W., \& Schmidt, M. 1964, ApJ, 140, 35

McLure, R. J., Kukula, M. J., Dunlop, J. S., et al. 1999, MNRAS, 308, 377

Meier, D. L. 1999, in Life cycles of radio galaxies, ed. Biretta et al., New Astron. Rev., in press [astro-ph/9908283]

Merritt, D. 1993, Science, 259, 1867

Michard, R. 1998a, A\&A, 334, 453

Michard, R. 1998b, A\&A, 335, 49

Michard, R., \& Marchal, J. 1994, A\&AS, 105, 481

Moderski, R., \& Sikora, M. 1996, MNRAS, 283, 854

Morganti, R., Killeen, N. E. B., \& Tadhunter, C. N. 1993, MNRAS, 263, 1023

Naab, T., Burkert, A., \& Hernquist, L. 1999, ApJ, 523, L133

Nieto, J.-L., \& Bender, R. 1989, A\&A, 215, 266

Nieto, J.-L., Bender, R., \& Surma, P. 1991, A\&A, 244, L37

Nilson, P. 1973, Uppsala General Catalog of Galaxies, Uppsala Astronomical Observatory, Uppsala

Owen, F. N., \& Laing, R. A. 1989, MNRAS, 238, 357

Owen, F. N., \& White, R. A. 1991, MNRAS, 249, 164

Peletier, R. F., Davies, R. L., Illingworth, G. D., et al. 1990, AJ, 100, 1091

Rees, M. J., Begelman, M. C., Blandford, R. D., \& Phinney, E. S. 1982, Nature, 295, 17

Sandage, A. 1961, The Hubble atlas of galaxies, Carnegie institution of Washington, Washington, DC

Sandage, A., \& Bedke, J. 1994, The Carnegie atlas of galaxies, Carnegie Institution of Washington, Washington DC

Sansom, A. E., Danziger, I. J., Ekers, R. D., et al. 1987, MNRAS, 229, 15

Savage, A., \& Wall, J. V. 1976, AJPS, 39, 39

Scheuer, P. A. G. 1992, in Extragalactic radio sources. From beams to jets, ed. J. Roland, H. Sol, \& G. Pelletier (Cambridge University Press), 368

Schombert, J. M. 1988, ApJ, 328, 475

Schweizer, F. 1980, ApJ, 237, 303

Shaver, P. A., Wall, J. V., Danziger, I. J., et al. 1983, MNRAS, 205, 819

Smith, E. P., \& Heckman, T. M. 1989a, ApJS, 69, 365

Smith, E. P., \& Heckman, T. M. 1989b, ApJ, 341, 658

Tonry, J. L. 1987, IAU Symp., 127, 89

Tremblay, B., \& Merritt, D. 1996, AJ, 111, 2243

Verdoes Kleijn, G. A., Baum, S. A., de Zeeuw, P. T., \& O'Dea, C. P. 2000, AJ, 118, 2592

Véron, P., \& Véron-Cetty, M. P. 1995, A\&A, 296, 315

Véron-Cetty, M. P., \& Woltjer, L. 1990, A\&A, 236, 69

Véron-Cetty, M. P., \& Woltjer, L. 1997, in Quasar hosts, ESO Astrophysics Symp., 27

Westerlund, B. E., \& Smith, L. F. 1966, AJP, 19, 181

Wilson, A. S., \& Colbert, E. J. M. 1995, ApJ, 438, 62

Zirbel, E. L. 1996, ApJ, 473, 713 